\title{
In-package MEMS-based thermal actuators for micro-assembly
}

\author{
V A Henneken ${ }^{1}$, M Tichem $^{1}$ and P M Sarro ${ }^{2}$ \\ ${ }^{1}$ Laboratory for Precision Manufacturing and Assembly, Mekelweg 2, 2628 CD Delft, \\ The Netherlands \\ ${ }^{2}$ Delft Institute of Microelectronics and Submicrontechnology (DIMES), \\ Delft University of Technology, The Netherlands \\ E-mail: v.a.henneken@tudelft.nl
}

Received 7 December 2005, in final form 9 March 2006

Published 10 May 2006

Online at stacks.iop.org/JMM/16/S107

\begin{abstract}
The paper describes the first fabrication and experimental results in the on-going development of MEMS-based electrothermal actuation devices for lateral $X Y$ positioning of an optical fibre to a laser diode chip to improve their coupling efficiency and reduce the overall packaging cost. The deflection performance of bulk silicon U- and V-beam thermal actuators with and without fibre loading has been experimentally determined. This is a part of an investigation of the feasibility of an alternative method of performing micro-assembly tasks, i.e. by means of product-internal assembly functions.
\end{abstract}

(Some figures in this article are in colour only in the electronic version)

\section{Introduction}

Performing micro-assembly tasks is technologically highly complicated due to the small part dimensions involved and the high-accuracy demands in positioning. The assembly cost of micro-parts can constitute a considerable portion of the total product cost (sometimes up to $80 \%$ ), due to the use of expensive machines and the involvement of delicate handwork. The micro-domain permits for the application of innovative methods of assembly. In [1], an overview has been presented of assembly methods as researched in the micro-mechanical engineering domain. The presented research focuses on the method of micro-assembly using product-internal assembly functions. The method is applied in a two-stage process. In the first stage, coarse positioning of components is achieved using product-external assembly functions, typically by a (semi-) automatic production machine or a human operator. The final, accurate positioning is performed on the basis of in-product assembly functions. Both coarse and fine positioning and fixation processes must be well adapted to each other to achieve an optimal overall result. The functions that apply for integration in the product are (1) controlled actuation of the component, (2) sensing the position of the component and (3) freezing of the component in the final position, see figure 1 .

The benefits that are aimed for are lower overall product costs and a higher product quality by reducing the amount of delicate micro-operations by human operators or expensive machines. For example, relaxing the placement tolerances from the sub- $\mu \mathrm{m}$ level to the $20 \mu \mathrm{m}$ level could potentially increase the throughput of a pick-and-place machine by more than an order of magnitude [2]. The added functionality needs to be low cost, since it remains part of the product during the operational life of the product. MEMS technology is considered to be a highly suitable technology for fabricating product-internal assembly functions, because of the small attainable feature sizes and very high accuracy. Due to the possibility of batch-wise processing it is potentially low cost, which is essential since the functionality remains part of the product after alignment. For these reasons, it is decided that at least the actuation functionality will be performed using MEMS technology. In the present investigation, it is the aim to research all aspects that influence how and under which circumstances MEMS-based product-internal functionality can be employed for the assembly of a particular product.

\section{Case: optoelectronic packaging}

The case considered for the investigation of the method of assembly using product-internal assembly functions is the alignment of a single-mode optical fibre to a laser diode, which is a challenging problem in optical telecommunication and sensing applications. This is embedded in the small 


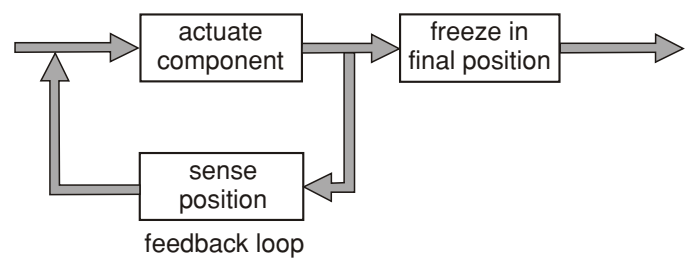

Figure 1. Product-internal assembly functions.

dimensions of the laser output waveguide (typically $2 \mu \mathrm{m} \times$ $0.4 \mu \mathrm{m}$ ) and the optical fibre (core diameter: $8 \mu \mathrm{m}$; overall fibre diameter: $125 \mu \mathrm{m}$ ). Alignment accuracy in the order of $\pm 0.1 \mu \mathrm{m}$ in the lateral dimension has to be maintained to achieve sufficient coupling efficiency over the economic lifetime of the system. Passive alignment and packaging techniques, which are preferred in optoelectronic applications for their intrinsic simplicity, can no longer be employed for these alignment requirements. Passive systems utilizing the so-called silicon waferboards or silicon optical benches (SiOBs) have reported alignment accuracies of 1-2 $\mu \mathrm{m}$ [2, 3], making active alignment a necessity in this case. The required alignment accuracy is very difficult to achieve by conventional methods in a reliable way. Very expensive machines or delicate manual handling are industrially employed for the necessary alignment and fixation steps, which last typically in the order of several minutes. To alleviate this need for expensive and slow macro assembly functionality, on-chip functionality for the final alignment of the optical fibre is attractive. The main challenge for such onchip alignment functionality is that of realizing the actuation of the fibre in the directions in which the coupling is most sensitive to inaccuracies, i.e. perpendicular to its optical axis. Previous devices have demonstrated multi-axis on-chip fibre alignment using various MEMS actuators [4-7]. However, these systems typically require complicated fabrication (LIGA [4]), specialized fibre preparation (permanent magnets [5], gold coating and grounding [6]) that limit their feasibility as a packaging option and/or very high-driving voltages to obtain acceptable actuation ranges [6, 7]. As opposed to this, the two-axis fibre positioning concepts proposed here do not have these specific requirements and can be realized using standard IC-compatible fabrication steps.

\section{Design considerations}

In this section, a short overview of the main design issues is presented for the first device concepts. Four concepts have been constructed for the actuation of the fibre tip in the $X Y$ directions perpendicular to the fibre. The main design considerations are presented here:

- desired travel range of 10-15 $\mu \mathrm{m}$ (based on possible coarse placement accuracy);

- forces in the $\mathrm{mN}$ range (to overcome stiffness fibre);

- minimum position resolution of $0.1 \mu \mathrm{m}$;

- small size (width preferably $<250 \mu \mathrm{m}$, standard fibre pitch used in industry);

- low complexity;

- sufficient robustness.

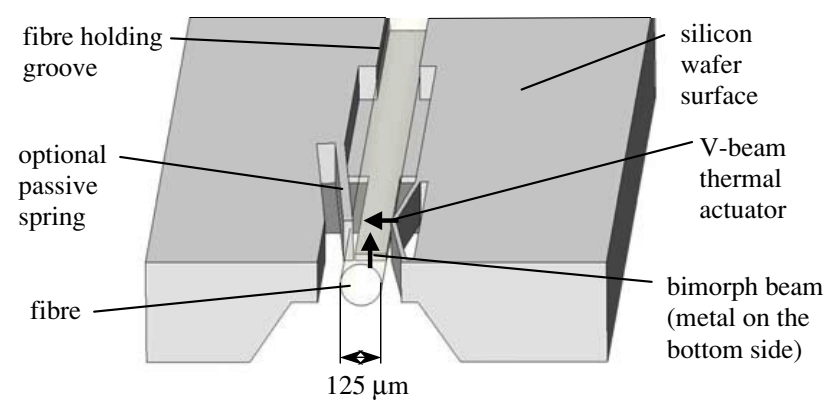

Figure 2. In-plane concept.

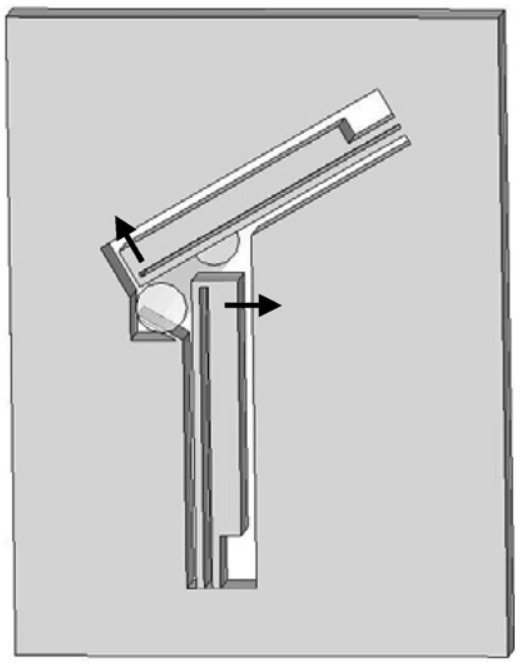

Figure 3. Through-plane concept.

Thermal expansion has been selected as an actuation principle for this application, since it possesses by far the best capabilities to achieve the necessary workload. The most commonly used actuation principles in the MEMS domain are electrostatic, piezoelectric, electromagnetic and electrothermal actuation [8]. For electrothermal actuation, displacements in excess of $20 \mu \mathrm{m}$ and forces as large as $40 \mathrm{mN}$ have been demonstrated [4]. Moreover, it generally consists of simple structures that can be made using proven technology with a limited number of fabrication steps.

There are three prevalent types of electrothermal actuators: the $V$-beam in which Joule heating accounts for the in-plane deformation of the structure (e.g. [9]), the U-beam for which the difference in heating between the narrow and the wide arm results in in-plane bending (e.g. [10]) and the bimorph beam for which a difference in the thermal expansion coefficient between two dissimilar materials upon heating results in out-of-plane bending of the beam (e.g. [11]). All of these have been quite extensively modelled, especially the U- and V-beam types, see for example [10-14], which is beneficial for the potential of successful applicability.

In figures 2 and 3 respectively one in-plane concept and one through-plane concept are presented.

In figure 2, the fibre is clamped between a V-beam structure and a passive spring. Horizontal motion of the fibre is performed by the V-beam and vertical motion by a bimorph beam with a metal layer at the bottom side. Figure 3 shows the 
fibre clamped between two U-beam structures and a passive spring. Upon heating the U-beams move sideways, thereby releasing the preload in the spring. The fibre can be inserted with both actuators in fully deflected state. Naturally, the overall device layout needs to be adapted to enable such a through-plane configuration.

\section{Device fabrication and testing}

In this section, the fabrication and testing results of the first demonstrator series are presented. To reduce the processing complexity, a limitation was made to in-plane actuation structures only (U- and V-beam actuators). Also, fine fixation functionality is not taken into consideration for the first demonstrators.

For the devices, bulk micromachining is employed since this renders the possibility of fabricating sufficiently robust structures for the considered application. The actuation devices may not be damaged during the coarse assembly step, and they should be able to produce adequate force to overcome the bending stiffness of the fibre and provide sufficient fibre tip travel range. Heating takes place by current flow in a thin doped polysilicon layer on the top surface. Diffusion of phosphorus has been used as a process to apply doping to the polysilicon layer. This has been selected to obtain relatively low-resistivity values in order to achieve sufficient power densities at manageable voltage levels. Using this process, a sheet resistance of $30 \Omega$ /square was obtained, compared to e.g. a lower limit of around $80 \Omega$ /square for boron implantation of polysilicon.

A considerable amount of out-of-plane stiffness is achieved through the use of a sufficiently large layer thickness, achieved by deep reactive ion etching (DRIE). This fabrication method is capable of providing structures having smooth, straight sidewalls perpendicular to the wafer surface with a large design freedom in the wafer plane. Attainable aspect ratios are large, depending on the minimum desired feature size. With feature sizes down to $20 \mu \mathrm{m}$, the maximum device layer thickness is safely set to $100-120 \mu \mathrm{m}$.

A schematic overview of the main fabrication steps is shown in figure 4. The starting substrate is a standard $525 \mu \mathrm{m}$ silicon wafer onto which a $300 \mathrm{~nm}$ oxide layer is thermally grown, followed by LPCVD of $300 \mathrm{~nm}$ low-stress silicon nitride (steps 1 and 2). Next, a $500 \mathrm{~nm}$ low-stress polysilicon layer is deposited using LPCVD, which is doped with phosphorus in a diffusion process, and subsequently patterned using a resist mask by means of dry etching (step 3). After this, a $300 \mathrm{~nm}$ TEOS oxide layer is deposited using LPCVD, which is similarly patterned using dry etching to form the openings for contacting the poly layer that is covered (step 4). Subsequently, aluminium is sputtered and patterned to form the electrical leads to the heaters (step 5), and the DRIE mask is created by plasma etching a PECVD oxide layer. This mask is used at the end of the flow to create the fibre grooves and actuators. The resist mask is left for scratch protection (step 6). On the backside, a $\mathrm{KOH}$ mask is created in the nitride-oxide layer, after which wet anisotropic etching is employed to form cavities until a substrate thickness of 100-120 $\mu \mathrm{m}$ remains (step 7). Finally, the resist scratch protect layer is removed, and DRIE is used to create the

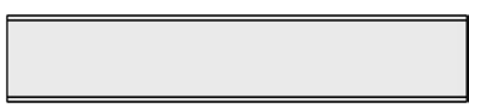

1. Thermal oxidation $300 \mathrm{~nm}$

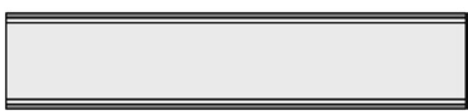

2. LPCVD SiN $300 \mathrm{~nm}$ low stress

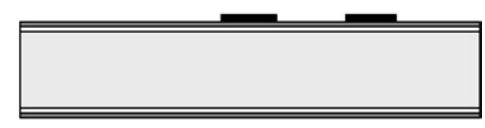

3. LPCVD polysilicon $500 \mathrm{~nm}$ low stress Phosphorus diffusion Poly dry etching

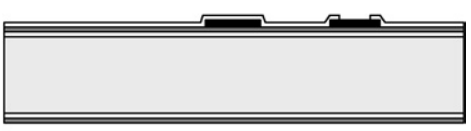

4. $3000 \AA ̊$ LPCVD TEOS oxide Dry etching contact openings

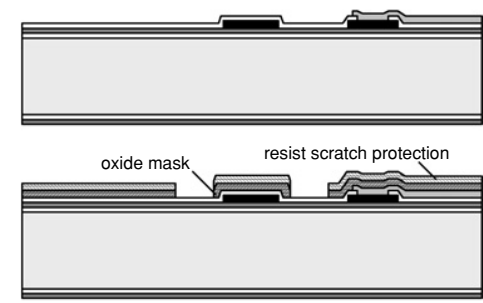

5. Aluminium sputtering $0,6 \mu \mathrm{m}$ Dry etching $\mathrm{Al}$ interconnects

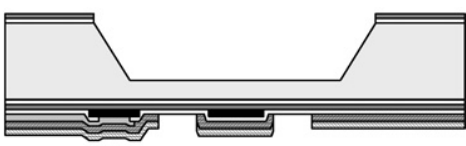

6. PECVD oxide + patterning to create DRIE mask Leave resist for scratch protection
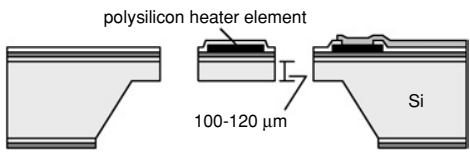

7. Backside wet etching using $\mathrm{KOH}$ mask Leave thickness 100-120 $\mu \mathrm{m}$

Figure 4. Fabrication sequence in-plane actuation structures.

actuator structures and fibre grooves, after which the oxide mask layer is removed (step 8).

The specific actuator structures used for experiments in this paper are presented in figure 5, together with their respective overall geometrical dimensions. The polysilicon layer, which is omitted here for clarity, is $4 \mu \mathrm{m}$ narrower on both sides than the structural beams to ensure that the polysilicon layer is fully covered (also on its sides) with the silicon dioxide cover layer to prevent the possible existence of undesired current paths.

Based on finite element modelling using ANSYS ${ }^{\mathrm{TM}}$ the expected free deflection at a $40 \mathrm{~V}$ load of the U-beam actuator is just below $15 \mu \mathrm{m}$ and for the V-beam actuator a little over $10 \mu \mathrm{m}$, see figures 6 and 7, respectively. Including fibre, these are both estimated to drop to around $10 \mu \mathrm{m}$ (the V-beam structure is significantly stiffer than the U-beam; hence, the difference in deflection decreases). The expected power needed for these deflections is $0.42 \mathrm{~W}$ for the U-beam and $0.64 \mathrm{~W}$ for the $\mathrm{V}$-beam structure, producing approximate peak temperatures locally of up to $500{ }^{\circ} \mathrm{C}$ and $600{ }^{\circ} \mathrm{C}$, respectively. Figure 8 provides numerical results of deflection and maximum temperature versus voltage for the range from 0 to $45 \mathrm{~V}$, for both the U-beam and V-beam thermal actuation structures. 


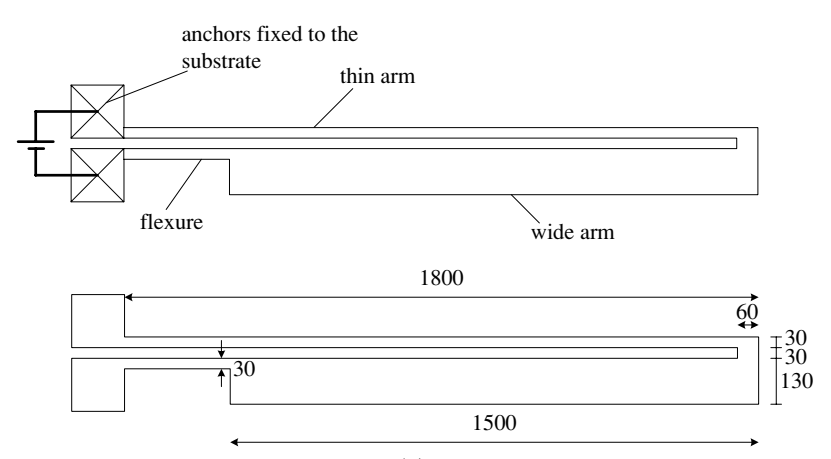

(a)

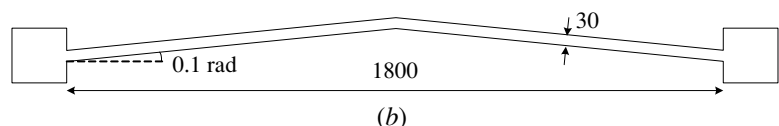

(b)

Figure 5. Schematic representation of $(a)$ the U-beam and $(b)$ the V-beam thermal actuation structures. The polysilicon layer follows the same geometry, is only $4 \mu \mathrm{m}$ narrower on each side.

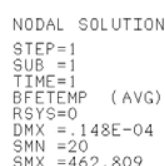

NN
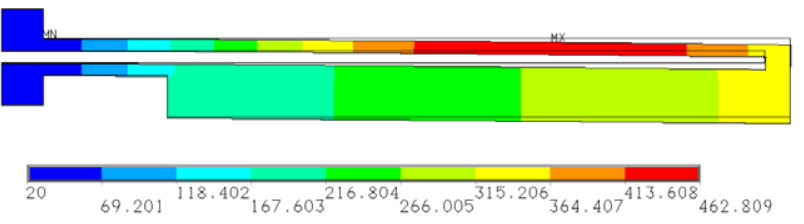

(a)

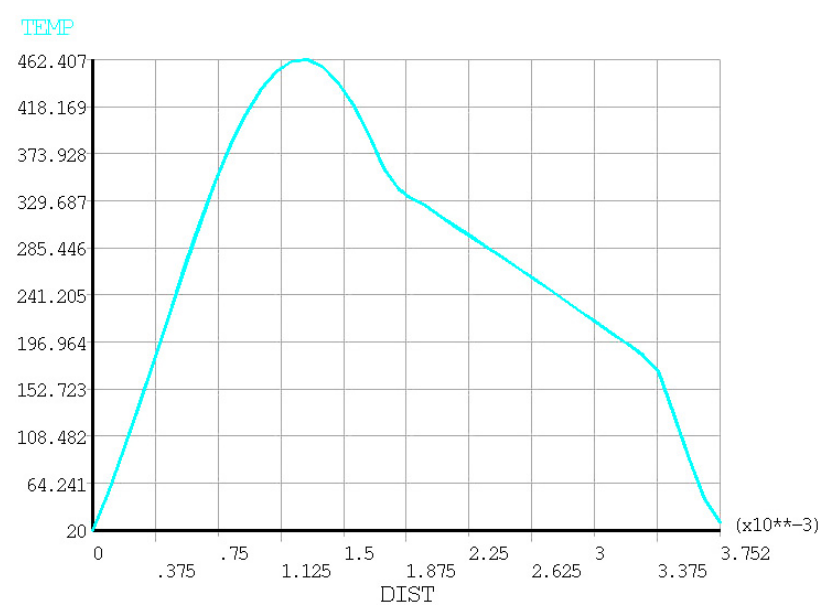

(b)

Figure 6. Finite element model of the U-beam thermal actuation structure at $40 \mathrm{~V}$ load $(a)$, and $(b)$ the matching temperature profile along the beams, starting from the anchor on the hot arm side.

The purpose of the finite element modelling was to be used as a virtual prototyping tool; the goal was to determine the overall dimensions needed for achieving the desired output performance. Instead of being as accurate as possible, the model should merely be sufficiently accurate

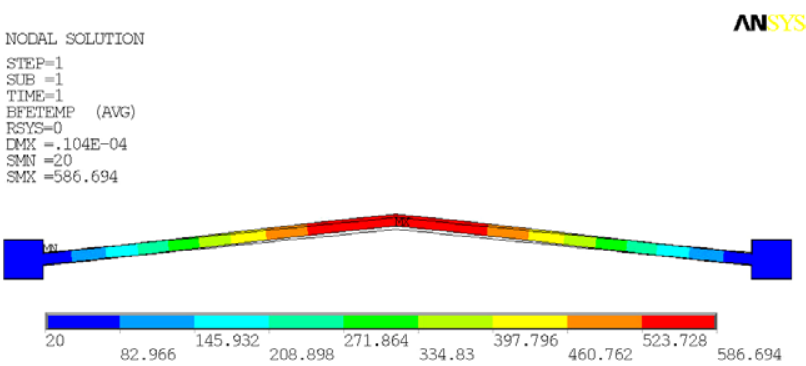

(a)

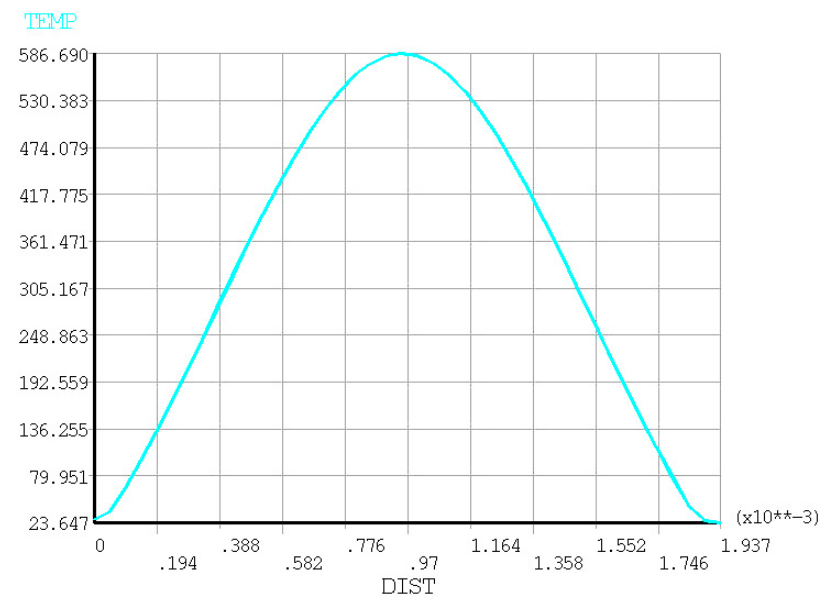

(b)

Figure 7. Finite element model of the V-beam thermal actuation structure at $40 \mathrm{~V}$ load $(a)$, and $(b)$ the matching temperature profile along the beam, starting from the anchor on the left side.

for this aim. For this reason, a number of simplifications were made. Convection, radiation and conduction through air were not taken into account. For the considered smallscale devices, convection and radiation play only a very minor role [10]; therefore, purely conductive heat transfer was assumed. Silicon is a very good heat conducting material; due to the relatively large cross-sectional beam area leading to the anchors (compared to surface micromachined actuators commonly employed) and since no substrate is closely present, the influence of conduction through the beam material is considered by far dominant over any conduction of heat through the surrounding air. Another simplification was made by excluding the thin polysilicon resistor layer (as well as the other thin deposited layers on top of the beams) from the models, and instead calculating with an equivalent electrical resistivity inside the bulk of the beams. This eliminates the possibility of predicting whether temperature gradients through the cross section of the actuators will be created of a magnitude sufficient to cause them to curl out of plane. This aspect was verified analytically. Mechanically, excluding the thin layers on the top surface has very little influence, in the order of the thickness ratio between these layers and the overall beam thickness $(\sim 1 \%)$. Young's modulus, the thermal expansion coefficient and the thermal conductivity were inserted into the models as temperature dependent.

The fabrication result can be observed in figures 9 and 10 . In figure 9, an overview image is provided for two individual U-beam actuators and one V-beam actuation structure after 


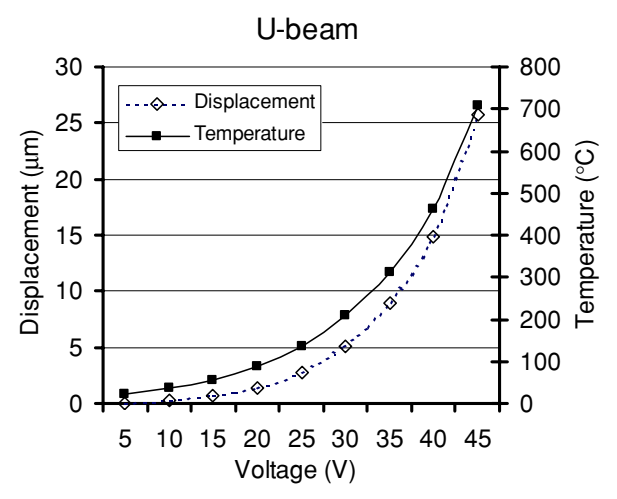

(a)

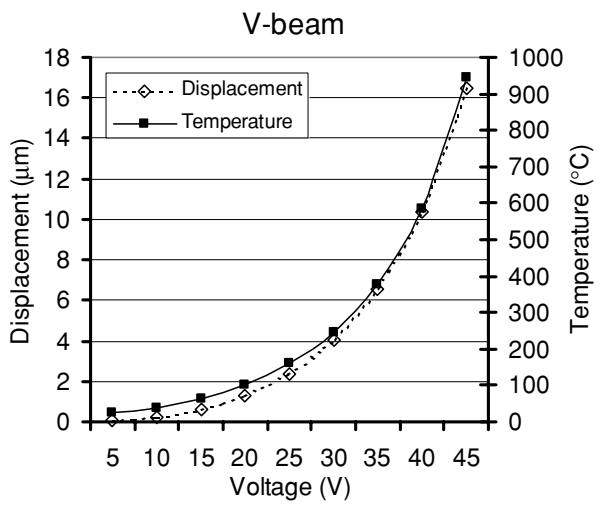

(b)

Figure 8. Modelled displacements and maximum temperatures at a voltage range from 0 to $45 \mathrm{~V},(a)$ for the U-beam and $(b)$ for the V-beam thermal actuation structures, respectively.

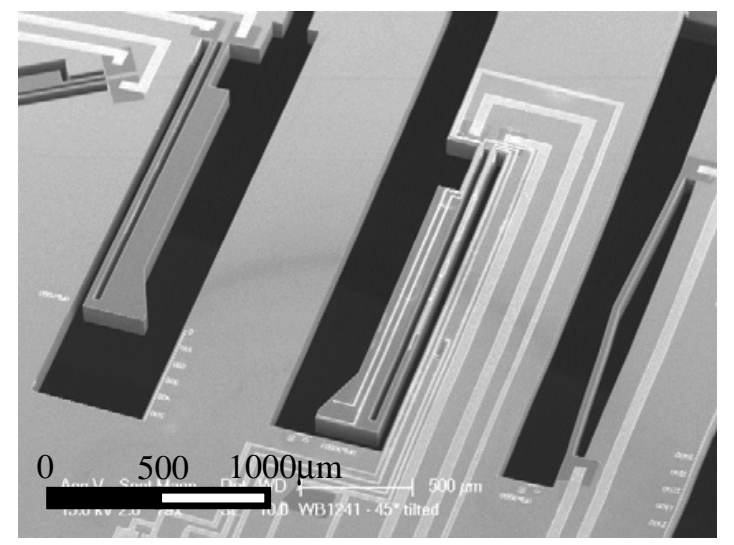

Figure 9. SEM image of two fabricated U-beam actuation structures (left) and one $\mathrm{V}$-beam actuator, at a $45^{\circ}$ viewing angle.

final release. Figure 10 shows a closeup of the end connection of the right U-beam actuator. Both images are taken from a $45^{\circ}$ viewing angle. It can be seen that the fabrication has rendered very smooth straight sidewalls without any observable under etch. The in-plane dimensions very accurately match the designed values. The cold actuator arm is equipped with an extra wide part at the top end in order for the fibre to have a well-defined contact area with the actuator structure. (The thin light-coloured aluminium lines on the top of the U-beam actuator are for possible temperature measurement purposes.)

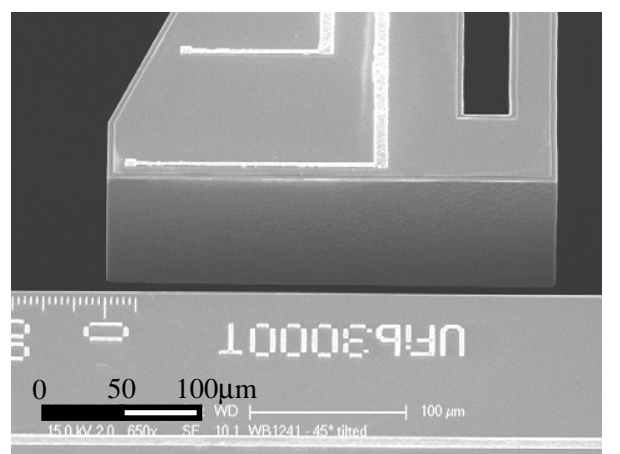

Figure 10. SEM closeup of the fabricated U-beam structure at a $45^{\circ}$ viewing angle.

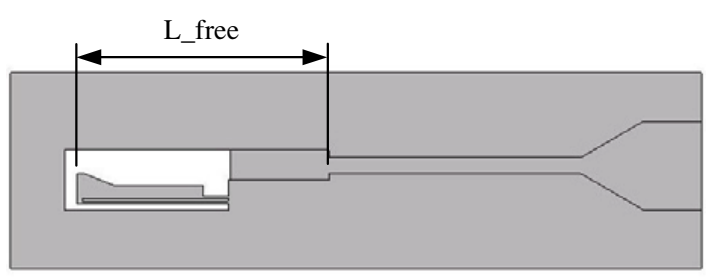

(a)

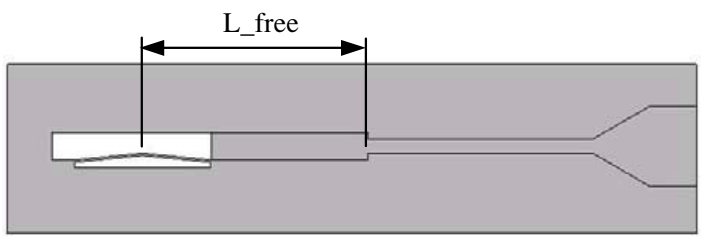

(b)

Figure 11. Schematic overall geometry of the U-beam structure (a) and of V-beam $(b)$, both with fibre holding groove. Indicated is the free length where the fibre is unsupported between groove and actuator contact area.

The actuator thickness was measured to be $130 \mu \mathrm{m}$, which is more than the $120 \mu \mathrm{m}$ originally aimed for, due to the fact that the wafer thickness of $525 \pm 25 \mu \mathrm{m}$ had not been measured before processing. Although this will give somewhat different actuation behaviour, this thickness variation is not critical and even though the $\mathrm{KOH}$ backside etching is a timed process the thickness could be controlled much more accurately if desired.

In order to be able to perform experiments both with and without fibre, a groove for holding a fibre has been devised extending from the actuator downwards. This fibre groove is designed to be $126 \mu \mathrm{m}$, which is just $1 \mu \mathrm{m}$ wider than the nominal fibre diameter. Between the actuatorfibre contact area and the narrow fibre groove, the fibre is sideways unsupported, see figure 11. This free length, which is denoted as $\mathrm{L}_{-}$free in the figure, is clearly defined and can be considered as the free length of a fibre clamped at the groove extremity. Both actuator structures have been fabricated with two different free fibre lengths $(2000$ and $3000 \mu \mathrm{m})$, since this has a significant effect on the stiffness to be overcome by the actuator (for $3000 \mu \mathrm{m}$ only around one-third of that for $2000 \mu \mathrm{m}$ ). The groove has been equipped with a taper on the outside for guiding the fibre when inserting from the side.

The actuator-fibre contact area and the fibre holding groove are placed exactly in line with each other, so when 


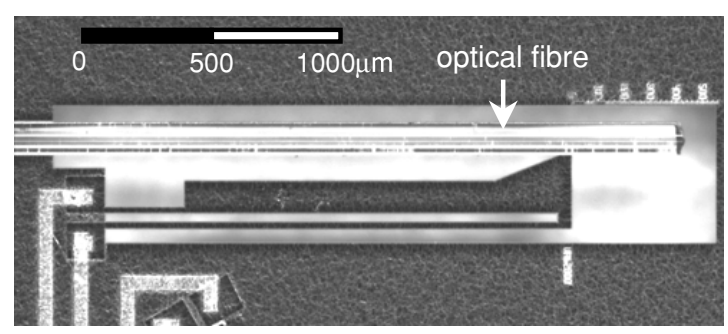

Figure 12. U-beam structure with fibre.

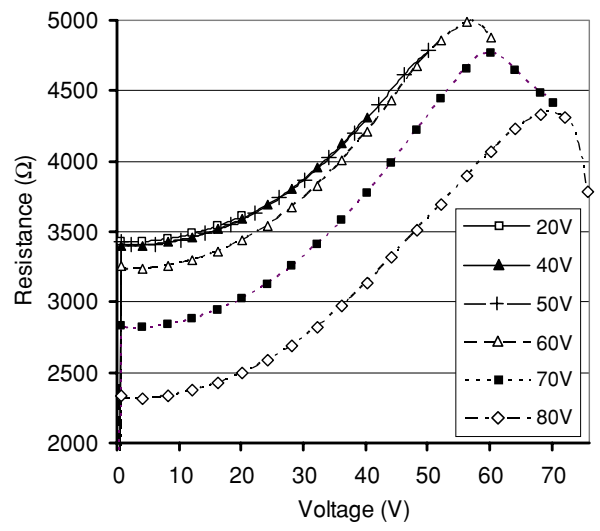

Figure 13. Voltage-resistance behaviour of the U-beam.

in off-state the fibre is nominally positioned just against the actuator. In reality the fibre ends are not always perfectly straight, and since an opposing passive spring was omitted in the design it is possible that the fibre is not in direct contact with the actuator when in off-state. Therefore for all measurements including fibre it was taken care of that the fibre at least made contact with the actuator. In practice this means that in offstate the fibre most of the times exerted a slight pretension force onto the actuator.

Inserting a fibre in either of the in-plane structures did not render unexpected difficulties. The manual handling operation is delicate, but no notable damage to the fibre or to the actuation structure was observed. Although also possible to be inserted from the top, it was particularly easy to insert a fibre from the side into the fibre groove. When inserting sideways no fibre buckling or obstructions due to friction effects were observed. See figure 12 for a top view of a U-beam structure with a fibre directly positioned against the actuator.

A first performance inspection of an $1800 \mu \mathrm{m}$ long U-beam actuator without fibre proved acceptable in accordance with the results of the modelled values. A linear increase in voltage from 0 to $40 \mathrm{~V}$ yielded an estimated maximum free end-effector displacement of over $10 \mu \mathrm{m}$. A colour change could be observed due to the temperature increase.

Subsequently, it was determined to which voltage level the actuators could be charged. For this purpose, a U-beam actuator was subjected to linear voltage ramps from zero subsequently to higher and higher end voltages. During this, the resistance value of the actuator was measured and plotted against the corresponding voltage level. The result of this test is presented in figure 13. For each graph, it can be observed

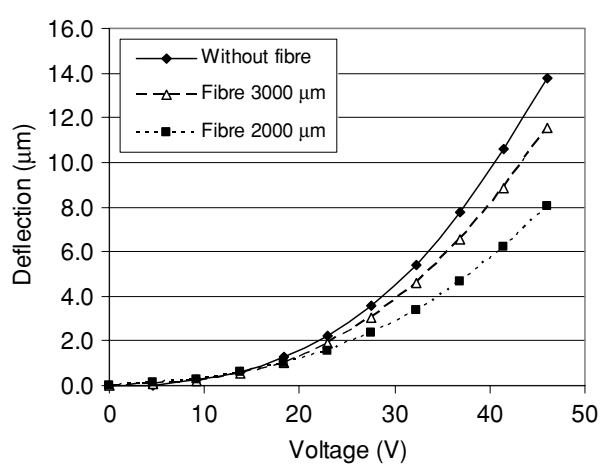

Figure 14. Voltage-deflection curve of the U-beam with and without fibre loading at two different clamping lengths.

that initially the resistance increases with increasing voltage. This is a known phenomenon for heavily doped polysilicon layers (e.g. [14]). Also it can be seen that the first three graphs (subsequently with the maximum voltages 20,40 and 50 V) for their common part closely correspond. Only the graph for the fourth linear increase (to $60 \mathrm{~V}$ ) notably starts to deviate from the previous three graphs. This can only be explained from a permanent change that has occurred in the previous cycle up to $50 \mathrm{~V}$. When completing the linear increase to $60 \mathrm{~V}$, it can be observed that the resistance reaches a certain maximum value, after which it starts to drop when proceeding to higher voltage levels. Even a further decrease in the resistance is observed for the following linear voltage ramp, indicating progressing change to the actuator structure. It can be seen that the top of the resistance curve has dropped to approximately the end resistance level of the previous curve. When attempting to increase the voltage in the subsequent cycle to $80 \mathrm{~V}$, the resistance level ultimately dropped very rapidly, which was accompanied by an uncontrolled current increase followed by a safety stop of the measurement equipment at approximately $75 \mathrm{~V}$. Just before reaching this value the actuation structure started emitting visible light at the thin arm, where the temperature was expected to reach the highest level. After this test, although the current path had been damaged significantly, it was not interrupted and the mechanical integrity of the overall structure was still intact. A possible failure mechanism of the actuators is the exceeding of the breakdown voltage in the dielectric layer between the polysilicon heater layer and the bulk silicon substrate layer. This dielectric layer is only around $0.6 \mu \mathrm{m}$ in thickness, and at high-driving voltages the electric field over the dielectric layer between polysilicon and substrate may become such that breakdown occurs, possibly also supported by elevated temperatures, thereby enabling the existence of parallel current paths through the lightly doped bulk silicon substrate, and by this lowering the overall resistance the electrical current encounters when travelling through the structure. For the V-beam structure, a similar behaviour was found, and a maximum voltage for both actuation structures for the remaining tests was set to $45 \mathrm{~V}$.

In figures 14 and 15 respectively the deflection behaviour of the U-beam as a function of applied voltage and of dissipated power is given. This is done both without fibre and with fibre clamped at two different lengths; at 2 and $3 \mathrm{~mm}$ from the 


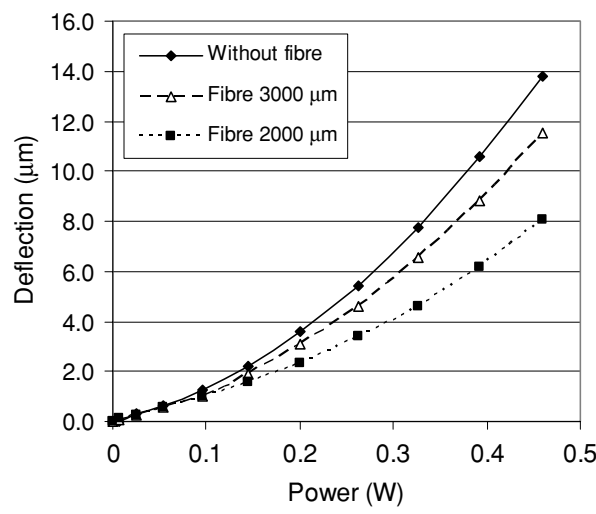

Figure 15. Power-deflection curve of the U-beam with and without fibre loading.

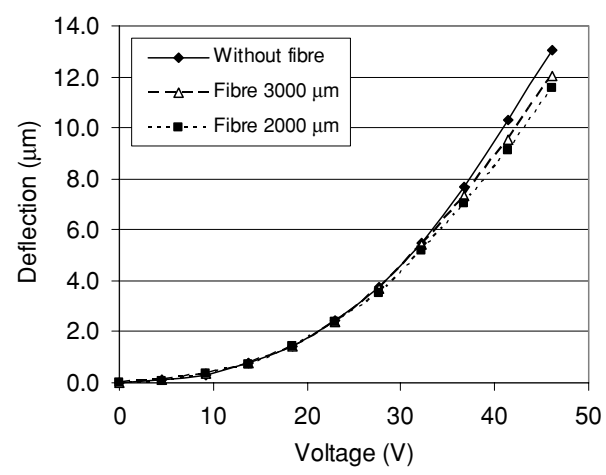

Figure 16. Voltage-deflection curve of the V-beam with and without fibre loading.

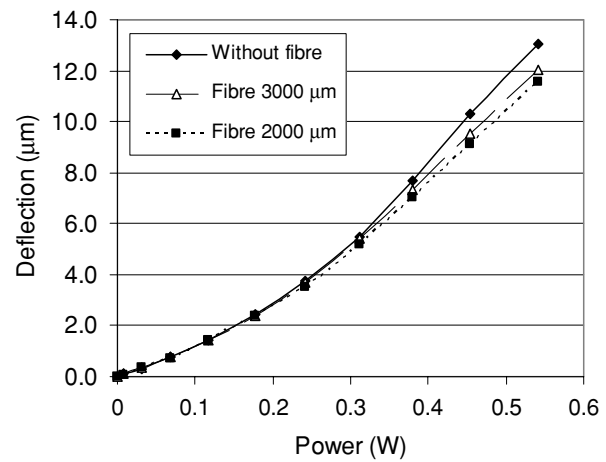

Figure 17. Power-deflection curve of the V-beam with and without fibre loading.

actuator tip. In figures 16 and 17 , the same is done for the V-beam actuator. The deflection measurements have been performed by means of software recognition using the inplane measurement functionality of a Polytec laser vibrometer, and are estimated to be approximately $50 \mathrm{~nm}$ accurate. As can be seen a significant difference in deflection between the cases with and without fibre is present, and also with the fibre at different clamping lengths. This can be explained from the comparable stiffness of the U-beam actuator and the fibre. At $2000 \mu \mathrm{m}$ clamping length, the fibre stiffness is close to the actuator stiffness, while at $3000 \mu \mathrm{m}$ clamping length the fibre stiffness is only around one-third of this value.

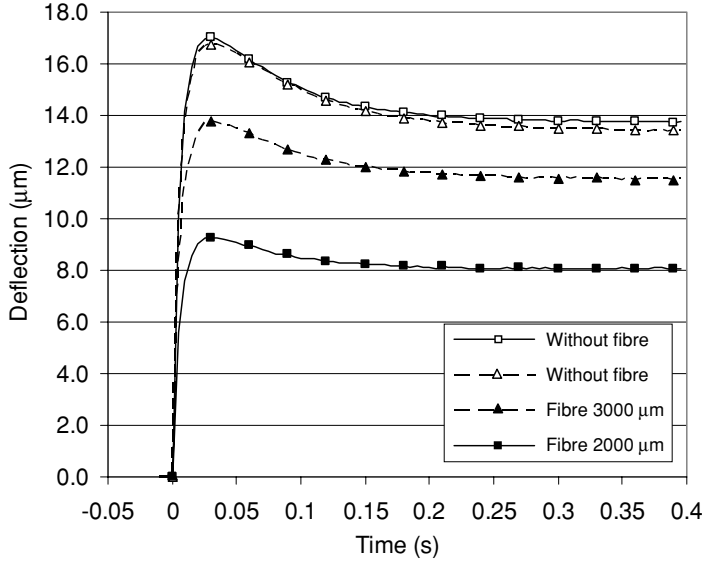

Figure 18. Step response of the U-beam structure with and without fibre loading. The test is performed two times without fibre.

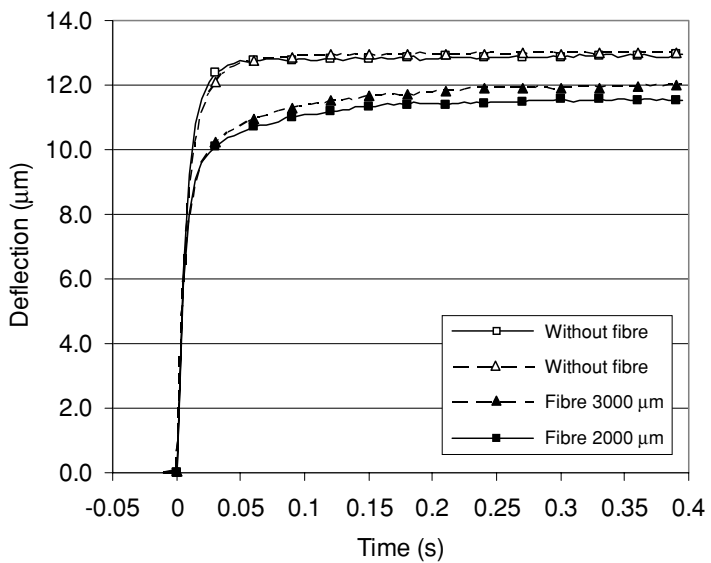

Figure 19. Step response of the V-beam structure with and without fibre loading.

Theoretically, the power-deflection behaviour is expected to be linear [10], but due to the considerable increase in resistance with increasing temperatures, the deviation from a linear curve is significant.

The V-beam actuator has a considerably higher stiffness than the fibre (and the U-beam actuator), and therefore the decrease in deflection due to the fibre loading is much smaller. Although the achievable deflection without fibre is lower for the V-beam than for the U-beam actuator, with a fibre clamped at $3000 \mu \mathrm{m}$ the deflection is comparable, and when the fibre is clamped at $2000 \mu \mathrm{m}$ the situation is reversed.

Finally, also using the Polytec laser vibrometer system the dynamic step responses of both the U- and V-beam actuators were obtained, again without and with fibre loading at two different clamping positions. These are presented in figures 18 and 19 , respectively. At time $t=0 \mathrm{~s}$, a voltage step signal of $45 \mathrm{~V}$ is applied to the actuation structure, after which the time-deflection behaviour is recorded. Both the U-beam and the V-beam actuators have been tested twice on different chips without fibre. As can be seen from the plotted graphs the results very closely match each other, indicating that the behaviour is quite reproducible.

For the U-beam actuator, a significant overshoot is observed, which decreases at increasing fibre load. The 


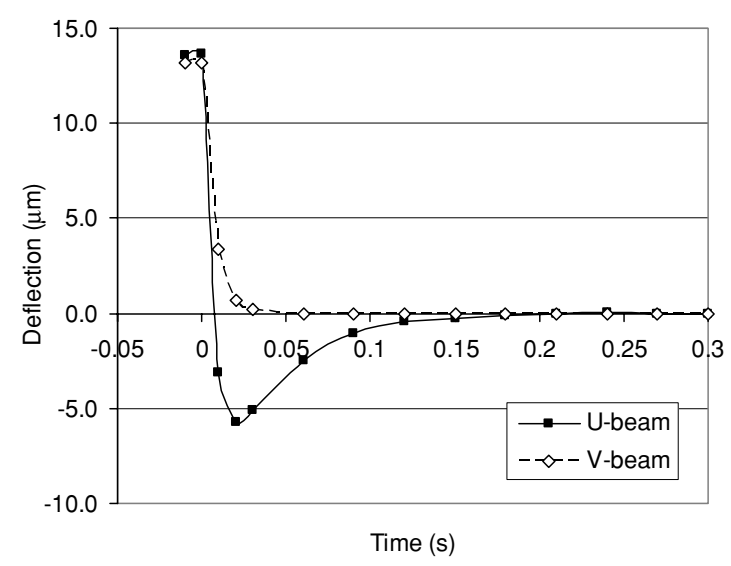

Figure 20. Cooling step response of the U- and V-beam structures.

V-beam in contrast exhibits no overshoot, but instead a gradual movement to its end position.

For this actuator, a slower reaching of its end position can be observed with fibre load. This can be explained by the thermal influence of the fibre onto the actuator. The contact between actuator and fibre is at the actuator location with the highest temperature. Due to leaking of heat at this location into the fibre the temperature profile over the actuator is altered notably. The low thermal diffusivity value $\alpha$ of the fibre compared to that of the actuator material is the cause for the somewhat longer time to establish a steady-state situation (glass has approximately 20 times lower thermal diffusivity than silicon, which indicates that glass adjusts much more slowly to changes in environment temperature). For the Ubeam actuator configuration, the situation is different, because the fibre-actuator contact area is quite some distance away from the hottest actuator location; therefore, it has much less effect on the (dynamic) deflection behaviour.

For both actuators after approximately $0.3 \mathrm{~s}$ the end position is reached to within $\pm 0.1 \mu \mathrm{m}$ (positioning criterion). Also shown is the cooling down behaviour, which, perhaps against intuition, was found to be even faster than the heating behaviour; typically within $0.2 \mathrm{~s}$ cooling down is achieved with a position stability of better than $\pm 0.1 \mu \mathrm{m}$ (see figure 20).

\section{Discussion}

The experimental results are in adequate agreement with the modelled values. Using the U- and V-beam thermal actuators free end-effector displacements of around 13-14 $\mu \mathrm{m}$ can be achieved at a voltage level of $45 \mathrm{~V}$. Including fibre the displacement reduces to $11-12 \mu \mathrm{m}$, except for the U-beam with $2000 \mu \mathrm{m}$ clamping length, for which the deflection drops to $8 \mu \mathrm{m}$. The power consumption at these deflections is approximately between 0.45 and $0.55 \mathrm{~W}$, with a typical heating time of around $0.3 \mathrm{~s}$.

For the U-beam, the modelling deviated in showing too high deflections at high voltages, while the power consumption was in quite good agreement. For the V-beam on the other hand, the modelled deflection was quite realistic, even at overestimated power levels. Overall, the deflections were lower than originally modelled, which may be due to the increasing electrical resistivity of the heavily doped polysilicon heater elements at elevated temperatures. The exact peak temperatures in the U- and V-beam actuators are unknown; no equipment was available to determine these parameters. Since the power levels during experimentation were lower than were established using the models, the maximum temperatures will also be lower than the modelled values. Therefore, it is estimated that at $45 \mathrm{~V}$ the maximum temperatures during the experiments have remained below $700{ }^{\circ} \mathrm{C}$.

The attainable travel range and position stability are sufficient for the fibre-chip coupling application, and also the control of the actuators can be acceptably fast. The V-beam is considered the best applicable actuation structure of the two, based on its high stiffness and its faster response without overshoot.

The power consumption is, as was expected, quite significant for both actuation structures, and therefore the actuators are not considered very suitable for continuous position control during product life. Instead, this indicates the desirability of fixation functionality to freeze the fibre relative to the other components after its actuation into the wanted position.

In contrast to intuition the local high temperatures do not give notable problems. In silicon, which is an excellent heat conducting material, the heat can be transported away very fast. If heat sinking is adequately taken care of, the overall device is not heated up significantly. For example at only a few hundred micrometers away from the U-beam anchors the temperature decreases to around ambient temperature, which is very beneficial for the device's overall position stability.

Although the polysilicon heater element is very thin and is located on the top surface of the beam structure, the time for the heat to penetrate downwards through the oxide and nitride layers to the single crystalline silicon appears to be very short compared to the time needed for the heat to reach the anchors. The temperature gradient over the vertical cross section of the actuators is sufficiently small, as can be inferred from the uniform deformation of the overall beam structure without any observable torsion or curling out of plane.

The influence of the fibre is found to be predominantly mechanical; due to its low thermal conductivity the heat loss through the fibre is very small compared to the heat produced in the actuators.

\section{Conclusions}

The first fabrication and experimental results are presented in the on-going development of MEMS-based electrothermal actuation devices for lateral $X Y$ positioning of an optical fibre to a laser diode chip. The experimental fibre positioning results using bulk silicon U- and V-beam thermal actuators are promising. The steady-state deflection output of the actuators with and without fibre, as well as their transient behaviour has been accurately determined using the available in-plane measurement capability. Including fibre loading in different configurations displacements between 8 and $12 \mu \mathrm{m}$ were obtained. The attainable travel range and position stability are sufficient for the considered application, and also the control of the actuators can be acceptably fast. The rather high-power 
consumptions reaching around $0.5 \mathrm{~W}$ make the actuators less suitable for continuous position control during product life.

\section{Acknowledgments}

This research belongs to the Delft Centre for Mechatronics and Microsystems of the TU Delft. It is funded by the Dutch government programme IOP Precision Engineering as part of the project IPT02310 Technologies for in-package optical fibre-chip coupling. Wouter Sassen is greatly acknowledged for his assistance in performing the experiments.

\section{References}

[1] Tichem M and Karpuschewski B 2002 Structuring of micro-assembly methods CD-ROM Proc. 33rd Int. Symp. on Robotics (Stockholm, 7-11 October)

[2] Schwab P, Bowen T, Perko R, Delen N, Goodrich J and Anderson R 2004 A high throughput optoelectronic module assembly process Proc. 54th IEEE Electronic Components and Technology Conf. (Las Vegas, NV, USA) pp 1475-8

[3] Li B, Wirz H and Sharon A 2005 Optimizing fiber coupling with a quasi-passive microoptical bench J. Microelectromech. Syst. 14 1339-46

[4] Haake J, Wood R and Duhler V 1998 In package micro aligner for fiber-optic packaging Proc. SPIE 3276 207-19
[5] Frank T 1998 Two-axis electrodynamic micropositioning devices J. Micromech. Microeng. 8 114-8

[6] Luetzelschwab M, Weiland D and Desmulliez M P Y 2005 Submicron alignment of a two-dimensional array of multiple single-mode fibers IEEE Photon. Technol. Lett. 17 2634-6

[7] Morgan B and Ghodssi R 2006 On-chip 2-axis optical fiber actuator using gray-scale technology Proc. MEMS'2006 pp 266-9

[8] Tabib-Azar M 1998 Microactuators (Dordrecht: Kluwer)

[9] Lott C D, McLain T W, Harb J N and Howell L L 2002 Modelling the thermal behaviour of a surface-micromachined linear-displacement thermomechanical microactuator Sensors Actuators A $101239-50$

[10] Hickey R, Sameoto D, Hubbard T and Kujath M 2003 Time and frequency response of two-arm micromachined thermal actuators J. Micromech. Microeng. 13 40-6

[11] Read B C, Bright V M and Comtois J H 1995 Mechanical and optical characterization of thermal microactuators fabricated in a CMOS process Proc. SPIE 2642 22-32

[12] Chiou J C and Lin W T 2004 Variable optical attenuator using a thermal actuator array with dual shutters Opt. Commun. 237 341-50

[13] Syms R R A 2002 Long-travel electrothermally driven resonant microactuators $J$. Micromech. Microeng. 12 211-8

[14] Huang Q-A and Lee N K S 1999 Analysis and design of polysilicon thermal flexure actuator J. Micromech. Microeng. 9 64-70 